\title{
Hopf bifurcation analysis in a predator-prey model with two time delays and stage structure for the prey
}

\author{
Miao Peng ${ }^{1}$ and Zhengdi Zhang ${ }^{1 *}$
}

${ }^{*}$ Correspondence:

dyzhang@ujs.edu.cn

${ }^{1}$ Faculty of Science, Jiangsu

University, Zhenjiang, P.R. China

\section{是 Springer}

\begin{abstract}
In this paper, a stage-structured predator-prey model with Holling type III functional response and two time delays is investigated. By analyzing the associated characteristic equation, its local stability and the existence of Hopf bifurcation with respect to both delays are studied. Based on the normal form method and center manifold theorem, the explicit formulas are derived to determine the direction of Hopf bifurcation and the stability of bifurcating period solutions. Finally, the effectiveness of theoretical analysis is verified via numerical simulations. This study may be helpful in understanding the behavior of ecological environment.
\end{abstract}

Keywords: Hopf bifurcation; Predator-prey model; Time delay; Stage structure; Local stability

\section{Introduction}

The dynamics of predator-prey models is one of important subjects in ecology and mathematical ecology, and many factors, such as time delay, disease, harvesting, functional response, etc., can affect it in the natural world.

In recent years, some researchers have discussed predator-prey models with these factors. Wangersky and Cunningham [1] considered a predator-prey system with time delay and studied the effect of time delay on the system. May [2] also analyzed the stability of vegetation-herbivore and vegetation-herbivore-carnivore systems with time delay. They found that a model with time delay could cause a stable equilibrium to become unstable and cause the population to fluctuate. Hu and $\mathrm{Li}$ [3] described a mathematical model dealing with a predator-prey system with disease in the prey, they discussed the properties of Hopf bifurcation. Banshidhar and Swarup [4] proposed a predator-prey model with harvesting where the top predator population is partially supported with alternative food. Their research showed that alternative food can prevent top predator extinction risk at higher harvesting effort. Yang [5] discussed the stability in a delayed diffusive predatorprey model with nonconstant death rate. Zhu et al. [6] studied a predator-prey model with time delay and square root response function. As we all know, non-smooth factor is used in mechanical engineering [7, 8]. In fact, non-smooth factor is also found in a predatorprey model. Chen and Huang [9] investigated a Filippov ratio-dependent predator-prey model to describe the effect on behavioral refuges caused by prey instinct anti-predator

(c) The Author(s) 2018. This article is distributed under the terms of the Creative Commons Attribution 4.0 International License (http://creativecommons.org/licenses/by/4.0/), which permits unrestricted use, distribution, and reproduction in any medium, provided you give appropriate credit to the original author(s) and the source, provide a link to the Creative Commons license, and indicate if changes were made. 
behavior. They discussed complete analysis of local and global dynamical behaviors of this model by Filippov qualitative theory. Peng et al. [10] proposed a hybrid control strategy in a predator-prey model. Their numerical simulation results showed that the hybrid controller is efficient in controlling a Hopf bifurcation.

In nature, there are many species whose individuals have a life history that can be divided into two stages: immature and mature. The stage structure reflects the species' activity ability and the difference about resistance to natural enemies in different growth period. It can affect the persistence and extinction of biological populations to various degree. Therefore, it is more practical to discuss the predator-prey model with this factor. Meng et al. [11] studied the stability and Hopf bifurcation in a three-species system with stage structure for the predator. Ref. $[12,13]$ also have paid great attention to discussing the effect with stage structure in predator-prey models. In [14], Wang et al. considered a delayed ratio-dependent predator-prey system with Holling type III functional response and stage structure for the predator:

$$
\begin{aligned}
& \dot{x}(t)=x(t)\left(r-a x\left(t-\tau_{1}\right)-\frac{a_{1} x(t) y_{2}(t)}{m y_{2}^{2}(t)+x^{2}(t)}\right), \\
& \dot{y}_{1}(t)=\frac{a_{2} x^{2}\left(t-\tau_{2}\right) y_{2}\left(t-\tau_{2}\right)}{m y_{2}^{2}\left(t-\tau_{2}\right)+x^{2}\left(t-\tau_{2}\right)}-r_{1} y_{1}(t)-D y_{1}(t), \\
& \dot{y}_{2}(t)=D y_{1}(t)-r_{2} y_{2}(t),
\end{aligned}
$$

where $x(t)$ represents the density of the prey population at time $t, y_{1}(t)$ and $y_{2}(t)$ describe the densities of the immature and the mature predator population at time $t$, respectively. The parameters $r, a, a_{1}, a_{2}, m, r_{1}, r_{2}$, and $D$ are positive constants in which $r$ represents the intrinsic growth rate of the prey, $a$ is the intraspecific competition rate of the prey, $a_{1}$ is the capturing rate, $a_{2} / a_{1}$ is the conversion rate of the mature predator, $m$ is the half capturing saturation constant, $r_{1}$ and $r_{2}$ are the death rates of the immature and the mature predator, respectively. $D$ denotes the rate at which the immature predator becomes the mature predator. $\tau_{1}$ is the feedback time delay of the prey, $\tau_{2}$ is the time delay due to the gestation of the mature predator. In [14], Wang et al. investigated the local stability of each of the feasible equilibria of the system and the related properties of Hopf bifurcation.

However, some predator species dislike hunting immature preys, or many immature preys are concealed in the caves or nests to keep from being attacked by the predators in the natural world. In this paper, based on the above discussions and motivated by the work of Wang et al. [14], we consider the following system with stage structure for the prey and two delays:

$$
\begin{aligned}
& \dot{x}_{1}(t)=a x_{2}(t)-b x_{1}(t)-r_{1} x_{1}(t)-c x_{1}(t) x_{1}\left(t-\tau_{1}\right)-\frac{a_{1} x_{1}^{2}(t) y(t)}{1+m x_{1}^{2}(t)}, \\
& \dot{x}_{2}(t)=b x_{1}(t)-r_{2} x_{2}(t), \\
& \dot{y}(t)=\frac{a_{2} x_{1}^{2}\left(t-\tau_{2}\right) y\left(t-\tau_{2}\right)}{1+m x_{1}^{2}\left(t-\tau_{2}\right)}-r y(t),
\end{aligned}
$$

where $x_{1}(t), x_{2}(t)$ describe the densities of the immature prey and the mature prey at time $t, y(t)$ represents the density of the predator at time $t$, respectively. $a$ is the birth rate of 
the immature prey, $b$ is the transformation rate from the immature prey to the mature one, $c$ is the intraspecific competition coefficient of the immature prey, $r_{1}, r_{2}, r$ denote the death rates of immature prey, mature prey, and predator. $a_{2} / a_{1}$ is the conversion rate of the predator, $\tau_{1}$ is the feedback time delay of the immature prey, and $\tau_{2}$ is the time delay due to the gestation of the predator. The rest of parameters $a_{1}, m$ are similar to those of model (1.1).

The organization of this paper is as follows. In Sect. 2, the local stability of the positive equilibrium and the existence of Hopf bifurcation for system (1.2) are studied. The direction of Hopf bifurcation and the stability of bifurcating periodic solutions are derived in Sect. 3. In Sect. 4, numerical simulations are carried out to illustrate the validity of the established results. Finally, a brief conclusion is given.

\section{Local stability and Hopf bifurcation}

From the viewpoint of biology, we only study the positive equilibrium of system (1.2). In this section, we shall discuss the local stability of a linearized system at the positive equilibrium and the existence of Hopf bifurcations for system (1.2).

It is easy to show that system (1.2) has a unique positive equilibrium $E^{*}\left(x_{1}^{*}, x_{2}^{*}, y^{*}\right)$, where

$$
\begin{aligned}
& x_{1}^{*}=\sqrt{\frac{r}{a_{2}-r m}}, \\
& x_{2}^{*}=\frac{b}{r_{2}} x_{1}^{*}, \\
& y^{*}=\frac{\left(a b-r_{1} r_{2}-b r_{2}-c r_{2} x_{1}^{*}\right)\left(1+m x_{1}^{* 2}\right)}{a_{1} r_{2} x_{1}^{*}}
\end{aligned}
$$

if the following conditions are satisfied:

$$
\text { (H1) } \quad a_{2}-r m>0, \quad a b-r_{1} r_{2}-b r_{2}-c r_{2} \sqrt{\frac{r}{a_{2}-r m}}>0 .
$$

Let $\bar{x}_{1}(t)=x_{1}(t)-x_{1}^{*}, \bar{x}_{2}(t)=x_{2}(t)-x_{2}^{*}, \bar{y}(t)=y(t)-y^{*}$ and still denote $\bar{x}_{1}(t), \bar{x}_{2}(t), \bar{y}(t)$, respectively. Using Taylor's expansion to expand system (1.2) at the positive equilibrium $E^{*}\left(x_{1}^{*}, x_{2}^{*}, y^{*}\right)$, we have

$$
\begin{aligned}
\dot{x}_{1}(t)= & a_{11} x_{1}(t)+a_{12} x_{2}(t)+a_{13} y(t)+b_{11} x\left(t-\tau_{1}\right) \\
& +\sum_{i+j+k+q \geq 2} f_{1}^{(i j k q)} x_{1}^{i}(t) x_{2}^{j}(t) y^{k} x_{1}^{q}\left(t-\tau_{1}\right), \\
\dot{x}_{2}(t)= & a_{21} x_{1}(t)+a_{22} x_{2}(t)+\sum_{i+j \geq 2} f_{2}^{(i j)} x_{1}^{i}(t) x_{2}^{j}(t), \\
\dot{y}(t)= & b_{31} x_{1}\left(t-\tau_{2}\right)+a_{33} y(t)+b_{33} y\left(t-\tau_{2}\right)+\sum_{i+j+k \geq 2} f_{3}^{(i j k)} y^{i}(t) x_{1}^{j}\left(t-\tau_{2}\right) y^{k}\left(t-\tau_{2}\right),
\end{aligned}
$$


where

$$
\begin{aligned}
& a_{11}=-r_{1}-c x_{1}^{*}-b-\frac{2 a_{1} x_{1}^{*} y^{*}}{\left[1+m\left(x_{1}^{*}\right)^{2}\right]^{2}}, \quad a_{12}=a, \\
& a_{13}=\frac{-a_{1}\left(x_{1}^{*}\right)^{2}}{1+m\left(x_{1}^{*}\right)^{2}}, \quad b_{11}=-c x_{1}^{*}, \\
& a_{21}=b, \quad a_{22}=-r_{2}, \quad b_{31}=\frac{2 a_{2} x_{1}^{*} y^{*}}{\left[1+m\left(x_{1}^{*}\right)^{2}\right]^{2}}, \\
& b_{33}=\frac{a_{2}\left(x_{1}^{*}\right)^{2}}{1+m\left(x_{1}^{*}\right)^{2}}, \quad a_{33}=-r . \\
& f_{1}^{(i j k q)}=\frac{1}{i ! j ! k ! q !} \frac{\partial^{i+j+k+q} f_{1}}{\partial x_{1}^{i}(t) \partial x_{2}^{j}(t) \partial y^{k}(t) \partial x^{q}\left(x-\tau_{1}\right)} \mid\left(x_{1}^{*}, x_{2}^{*}, y^{*}\right), \\
& f_{2}^{(i j)}=\frac{1}{i ! j !} \frac{\partial^{i+j} f_{2}}{\partial x_{1}^{i}(t)} \mid\left(x_{1}^{j}, x_{2}^{*}, y^{*}\right), \\
& f_{3}^{(i j k)}=\frac{1}{i ! j ! k !} \frac{\partial^{i+j+k} f_{3}}{\partial y^{i}(t) \partial x_{1}^{j}\left(t-\tau_{2}\right) \partial y^{k}\left(t-\tau_{2}\right)} \mid\left(x_{1}^{*}, x_{2}^{*}, y^{*}\right), \\
& f_{1}=a x_{2}(t)-b x_{1}(t)-r_{1} x_{1}(t)-c x_{1}(t) x_{1}\left(t-\tau_{1}\right)-\frac{a_{1} x_{1}^{2}(t) y(t)}{1+m x_{1}^{2}(t)}, \\
& f_{2}=b x_{1}(t)-r_{2} x_{2}(t), \quad f_{3}=\frac{a_{2} x_{1}^{2}\left(t-\tau_{2}\right) y\left(t-\tau_{2}\right)}{1+m x_{1}^{2}\left(t-\tau_{2}\right)}-r y(t) .
\end{aligned}
$$

Then we obtain the linearized system of system (2.1) as follows:

$$
\begin{aligned}
& \dot{x}_{1}(t)=a_{11} x_{1}(t)+a_{12} x_{2}(t)+a_{13} y(t)+b_{11} x\left(t-\tau_{1}\right), \\
& \dot{x}_{2}(t)=a_{21} x_{1}(t)+a_{22} x_{2}(t), \\
& \dot{y}(t)=b_{31} x_{1}\left(t-\tau_{2}\right)+a_{33} y(t)+b_{33} y\left(t-\tau_{2}\right) .
\end{aligned}
$$

Therefore, the corresponding characteristic equation of system (2.2) is given by

$$
\begin{aligned}
\lambda^{3} & +m_{2} \lambda^{2}+m_{1} \lambda+m_{0}+\left(n_{2} \lambda^{2}+n_{1} \lambda+n_{0}\right) e^{-\lambda \tau_{1}} \\
& +\left(p_{2} \lambda^{2}+p_{1} \lambda+p_{0}\right) e^{-\lambda \tau_{2}}+\left(q_{1} \lambda+q_{0}\right) e^{-\lambda\left(\tau_{1}+\tau_{2}\right)}=0,
\end{aligned}
$$

where

$$
\begin{aligned}
& m_{0}=a_{12} a_{21} a_{33}-a_{11} a_{22} a_{33}, \quad m_{1}=a_{11} a_{22}+a_{22} a_{33}+a_{11} a_{33}-a_{12} a_{21}, \\
& m_{2}=-\left(a_{11}+a_{22}+a_{33}\right), \\
& n_{0}=-b_{11} a_{22} a_{33}, \quad n_{1}=b_{11}\left(a_{22}+a_{33}\right), \quad n_{2}=-b_{11}, \\
& p_{0}=a_{13} a_{22} b_{31}+a_{12} a_{21} b_{33}-a_{11} a_{22} a_{33}, \\
& p_{1}=a_{11} b_{33}+a_{22} b_{33}-a_{13} b_{31}, \quad p_{2}=-b_{33}, \\
& q_{0}=-a_{22} b_{11} b_{33}, \quad q_{1}=b_{11} b_{33} .
\end{aligned}
$$

In order to investigate the root distribution of the transcendental Eq. (2.3), the result of Ruan and Wei [15] is introduced here. 
Lemma 2.1 For the transcendental equation

$$
\begin{aligned}
p\left(\lambda, e^{-\lambda \tau_{1}}, \ldots, e^{-\lambda \tau_{m}}\right)= & \lambda^{n}+p_{1}^{(0)} \lambda^{n-1}+\cdots+p_{n-1}^{(0)} \lambda+p_{n}^{(0)} \\
& +\left[p_{1}^{(1)} \lambda^{n-1}+\cdots+p_{n-1}^{(1)} \lambda+p_{n}^{(1)}\right] e^{-\lambda \tau_{1}}+\cdots \\
& +\left[p_{1}^{(m)} \lambda^{n-1}+\cdots+p_{n-1}^{(m)} \lambda+p_{n}^{(m)}\right] e^{-\lambda \tau_{m}} \\
= & 0
\end{aligned}
$$

as $\left(\tau_{1}, \tau_{2}, \tau_{3}, \ldots, \tau_{m}\right)$ vary, the sum of orders of the zeros of $p\left(\lambda, e^{-\lambda \tau_{1}}, \ldots, e^{-\lambda \tau_{m}}\right)$ in the open right half plane can change, and only a zero appears on or crosses the imaginary axis.

Next, we will consider the following four cases.

Case 1: $\tau_{1}=\tau_{2}=0$, the characteristic Eq. (2.3) reduces to

$$
\lambda^{3}+m_{12} \lambda^{2}+m_{11} \lambda+m_{10}=0
$$

where $m_{10}=m_{0}+n_{0}+p_{0}+q_{0}, m_{11}=m_{1}+n_{1}+p_{1}, m_{12}=m_{2}+n_{2}+p_{2}$.

It is not difficult to verify that $m_{10}>0, m_{12}>0$. Thus, all the roots of Eq. (2.4) have negative real parts if the following condition holds:

(H11) $\quad m_{11} m_{12}>m_{10}$.

Namely, the equilibrium point $E^{*}\left(x_{1}^{*}, x_{2}^{*}, y^{*}\right)$ is locally asymptotically stable when condition (H11) is satisfied.

Case 2: $\tau_{1}=0, \tau_{2}>0$. Equation (2.3) becomes

$$
\lambda^{3}+m_{22} \lambda^{2}+m_{21} \lambda+m_{20}+\left(p_{22} \lambda^{2}+p_{21} \lambda+p_{20}\right) e^{-\lambda \tau_{2}}=0,
$$

where $m_{20}=m_{0}+n_{0}, m_{21}=m_{1}+n_{1}, m_{22}=m_{2}+n_{2}, p_{20}=p_{0}+q_{0}, p_{21}=p_{1}+q_{1}, p_{22}=p_{2}$.

Let $i \omega_{2}\left(\omega_{2}>0\right)$ be a root of Eq. (2.5), it follows that

$$
\begin{aligned}
& p_{21} \omega_{2} \sin \omega_{2} \tau_{2}+\left(p_{20}-p_{22} \omega_{2}^{2}\right) \cos \omega_{2} \tau_{2}=m_{22} \omega_{2}^{2}-m_{20}, \\
& p_{21} \omega_{2} \cos \omega_{2} \tau_{2}-\left(p_{20}-p_{21} \omega_{2}^{2}\right) \sin \omega_{2} \tau_{2}=\omega_{2}^{3}-m_{21} \omega_{2},
\end{aligned}
$$

which leads to

$$
\omega_{2}^{6}+e_{22} \omega_{2}^{4}+e_{21} \omega_{2}^{2}+e_{20}=0
$$

where $e_{20}=m_{20}^{2}-p_{20}^{2}, e_{21}=m_{21}^{2}-2 m_{20} m_{22}-p_{21}^{2}+2 p_{20} p_{22}, e_{22}=m_{22}^{2}-2 m_{21}-p_{22}^{2}$.

Let $\omega_{2}^{2}=v_{2}$, then Eq. (2.7) can be written as

$$
v_{2}^{3}+e_{22} v_{2}^{2}+e_{21} v_{2}+e_{20}=0
$$

Denote

$$
f_{1}\left(v_{2}\right)=v_{2}^{3}+e_{22} v_{2}^{2}+e_{21} v_{2}+e_{20}
$$


Since $f_{1}(0)=e_{20}, \lim _{v_{2} \rightarrow+\infty} f_{1}\left(v_{2}\right)=+\infty$, and from Eq. (2.9), we have

$$
f_{1}^{\prime}\left(v_{2}\right)=3 v_{2}^{2}+2 e_{22} v_{2}+e_{21}
$$

After discussion about the roots of Eq. (2.10) similar to that in [16], we have the following lemma.

Lemma 2.2 For the polynomial Eq. (2.8), we have the following results:

(1) If

$$
(\mathrm{H} 21) \quad e_{20} \geq 0, \quad \Delta=e_{22}^{2}-3 e_{21} \leq 0
$$

holds, then Eq. (2.8) has no positive root;

(2) If

$$
(\mathrm{H} 22) \quad e_{20} \geq 0, \quad \Delta=e_{22}^{2}-3 e_{21}>0, \quad v_{2}^{*}=\frac{-e_{21}+\sqrt{\Delta}}{3}>0, \quad f_{1}\left(v_{2}^{*}\right) \leq 0,
$$

or

(H23) $\quad e_{20}<0$

holds, then Eq. (2.8) has a positive root.

Suppose that Eq. (2.8) has positive roots. Without loss of generality, we assume that it has three positive roots, which are denoted by $v_{21}, v_{22}$, and $v_{23}$. Then Eq. (2.7) has three positive roots $\omega_{2 k}=\sqrt{\nu_{2 k}}, k=1,2,3$. The corresponding critical value of time delay $\tau_{2 k}^{(j)}$ is

$$
\tau_{2 k}^{(j)}=\frac{1}{\omega_{2 k}} \arccos \left\{\frac{A_{24} \omega_{2 k}^{4}+A_{22} \omega_{2 k}^{2}+A_{20}}{B_{24} \omega_{2 k}^{4}+B_{22} \omega_{2 k}^{2}+B_{20}}\right\}+\frac{2 \pi j}{\omega_{2 k}}, \quad k=1,2,3 ; j=0,1,2, \ldots,
$$

where $A_{20}=-m_{20} p_{20}, A_{22}=m_{22} p_{20}+m_{20} p_{22}-m_{21} p_{21}, A_{24}=p_{21}-m_{22} p_{22}, B_{20}=p_{20}^{2}, B_{22}=$ $p_{21}^{2}-2 p_{20} p_{22}, B_{24}=p_{22}^{2}$.

Thus $\pm \omega_{2 k}$ is a pair of purely imaginary roots of Eq. (2.5) with $\tau_{2}=\tau_{2 k}^{(j)}$, and let $\tau_{20}=$ $\min _{k \in\{1,2,3\}}\left\{\tau_{2 k}^{(0)}\right\}, \omega_{20}=\omega_{2 k_{0}}$.

\section{Lemma 2.3 Suppose that}

$$
\text { (H24) } f_{1}^{\prime}\left(\omega_{20}^{2}\right) \neq 0 \text {, }
$$

then the following transversality condition holds:

$$
\left\{\frac{d(\operatorname{Re} \lambda)}{d \tau_{2}}\right\}_{\lambda=i \omega_{20}} \neq 0
$$

Proof Differentiating Eq. (2.5) with respect to $\tau_{2}$, and noticing that $\lambda$ is a function of $\tau_{2}$, we obtain

$$
\left(\frac{d \lambda}{d \tau_{2}}\right)^{-1}=-\frac{3 \lambda^{2}+2 m_{22} \lambda+m_{21}}{\lambda\left(\lambda^{3}+m_{22} \lambda^{2}+m_{21} \lambda+m_{20}\right)}+\frac{2 p_{22} \lambda+p_{21}}{\lambda\left(p_{22} \lambda^{2}+p_{21} \lambda+n_{20}\right)}-\frac{\tau_{1}}{\lambda},
$$


which leads to

$$
\begin{aligned}
\operatorname{Re}\left(\frac{d \lambda}{d \tau_{2}}\right)^{-1}= & \operatorname{Re}\left(-\frac{3 \lambda^{2}+2 m_{22} \lambda+m_{21}}{\lambda\left(\lambda^{3}+m_{22} \lambda^{2}+m_{21} \lambda+m_{20}\right)}\right)_{\lambda=i \omega_{20}} \\
& +\operatorname{Re}\left(\frac{2 p_{22} \lambda+p_{21}}{\lambda\left(p_{22} \lambda^{2}+p_{21} \lambda+p_{20}\right)}\right)_{\lambda=i \omega_{20}} \\
= & \frac{3 \omega_{20}^{4}+2\left(m_{22}^{2}-2 m_{21}\right) \omega_{20}^{2}+m_{21}^{2}-2 m_{20} m_{22}}{\left(\omega_{20}^{3}-m_{21} \omega_{20}\right)^{2}+\left(m_{20}-m_{22} \omega_{20}^{2}\right)^{2}}-\frac{2 p_{22}^{2} \omega_{20}^{2}+p_{21}^{2}-2 p_{20} p_{22}}{\left(p_{22} \omega_{20}^{2}-p_{20}\right)^{2}+p_{21}^{2} \omega_{20}^{2}}
\end{aligned}
$$

From Eq. (2.6), we have

$$
\left(\omega_{20}^{3}-m_{21} \omega_{20}\right)^{2}+\left(m_{20}-m_{22} \omega_{20}^{2}\right)^{2}=\left(p_{22} \omega_{20}^{2}-p_{20}\right)^{2}+p_{21}^{2} \omega_{20}^{2}
$$

Noting that $\left\{\frac{d(\operatorname{Re} \lambda)}{d \tau_{1}}\right\}_{\lambda=i \omega_{20}}$ and $\left\{\operatorname{Re}\left(\frac{d \lambda}{d \tau_{1}}\right)^{-1}\right\}_{\lambda=i \omega_{20}}$ have the same sign, then

$$
\begin{aligned}
\operatorname{sign}\left\{\frac{d(\operatorname{Re} \lambda)}{d \tau_{1}}\right\}_{\lambda=i \omega_{20}} & =\operatorname{sign}\left\{\operatorname{Re}\left(\frac{d \lambda}{d \tau_{1}}\right)^{-1}\right\}_{\lambda=i \omega_{20}}=\frac{3\left(\omega_{20}^{2}\right)^{2}+2 e_{22} \omega_{20}^{2}+e_{21}}{p_{21}^{2} \omega_{20}^{2}+\left(p_{20}-p_{22} \omega_{20}^{2}\right)^{2}} \\
& =\frac{f_{1}^{\prime}\left(\omega_{20}^{2}\right)}{p_{21}^{2} \omega_{20}^{2}+\left(p_{20}-p_{22} \omega_{20}^{2}\right)^{2}} \\
& \neq 0 .
\end{aligned}
$$

It follows that $\left\{\frac{d(\operatorname{Re} \lambda)}{d \tau_{2}}\right\}_{\lambda=i \omega_{20}} \neq 0$ and the proof is complete.

By Lemmas 2.1-2.3, and combining the Hopf bifurcation theorem [17-19], we have the following results.

Theorem 2.1 For system (1.2), $\tau_{1}=0$.

(1) If ( $\mathrm{H} 21)$ holds, then the positive equilibrium $E^{*}\left(x_{1}^{*}, x_{2}^{*}, y^{*}\right)$ is asymptotically stable for all $\tau_{2} \geq 0$.

(2) If $(\mathrm{H} 22)$ or $(\mathrm{H} 23)$ and $(\mathrm{H} 24)$ hold, then the positive equilibrium $E^{*}\left(x_{1}^{*}, x_{2}^{*}, y^{*}\right)$ is asymptotically stable for all $\tau_{2} \in\left[0, \tau_{20}\right)$ and unstable for $\tau_{2}>\tau_{20}$. Furthermore, system (1.2) undergoes a Hopf bifurcation at the positive equilibrium $E^{*}\left(x_{1}^{*}, x_{2}^{*}, y^{*}\right)$ when $\tau_{2}=\tau_{20}$.

Case 3: $\tau_{1}=\tau_{2}=\tau \neq 0$. Equation (2.3) reduces to

$$
\lambda^{3}+m_{32} \lambda^{2}+m_{31} \lambda+m_{30}+\left(n_{32} \lambda^{2}+n_{31} \lambda+n_{30}\right) e^{-\lambda \tau}+\left(q_{31} \lambda+q_{30}\right) e^{-2 \lambda \tau}=0,
$$

where $m_{30}=m_{0}, m_{31}=m_{1}, m_{32}=m_{2}, n_{30}=n_{0}+p_{0}, n_{31}=n_{1}+p_{1}, n_{32}=n_{2}+p_{2}, q_{30}=q_{0}$, $q_{31}=q_{1}$.

Multiplying by $e^{\lambda \tau}$, Eq. (2.15) becomes

$$
\left(\lambda^{3}+m_{32} \lambda^{2}+m_{31} \lambda+m_{30}\right) e^{\lambda \tau}+\left(n_{32} \lambda^{2}+n_{31} \lambda+n_{30}\right)+\left(q_{41} \lambda+q_{40}\right) e^{-\lambda \tau}=0 .
$$


Let $i \omega(\omega>0)$ be the root of Eq. (2.16), and separate the real and imaginary parts, we have

$$
\begin{aligned}
& E_{31} \sin \omega \tau+E_{32} \cos \omega \tau=E_{35}, \\
& E_{33} \cos \omega \tau+E_{34} \sin \omega \tau=E_{36},
\end{aligned}
$$

where $E_{31}=-m_{31} \omega+\omega^{3}+q_{31} \omega, E_{32}=m_{30}-m_{32} \omega^{2}+q_{30}$,

$$
\begin{aligned}
& E_{33}=m_{31} \omega-\omega^{3}+q_{31} \omega, \quad E_{34}=m_{30}-m_{32} \omega^{2}-q_{30}, \\
& E_{35}=n_{32} \omega^{2}-n_{30}, \quad E_{36}=-n_{31} \omega .
\end{aligned}
$$

It follows that

$$
\sin \omega \tau=\frac{A_{35} \omega^{5}+A_{33} \omega^{3}+A_{31} \omega}{\omega^{6}+B_{34} \omega^{4}+B_{32} \omega^{2}+B_{30}}, \quad \cos \omega \tau=\frac{A_{34} \omega^{4}+A_{32} \omega^{4}+A_{30}}{\omega^{6}+B_{34} \omega^{4}+B_{32} \omega^{2}+B_{30}}
$$

where

$$
\begin{aligned}
& A_{30}=\left(q_{30}-m_{30}\right) n_{30}, \quad A_{31}=\left(m_{31}+q_{31}\right) n_{30}-\left(m_{30}+q_{30}\right) n_{31}, \\
& A_{32}=\left(m_{30}-q_{30}\right) n_{32}-m_{31} n_{31}+m_{32} n_{30}+q_{31} n_{31}, \\
& A_{33}=m_{32} n_{31}-n_{30}-m_{31} n_{32}-q_{31} n_{32}, \\
& A_{34}=n_{31}-m_{32} n_{32}, \quad A_{35}=n_{32}, \quad B_{30}=m_{30}^{2}-q_{30}^{2}, \\
& B_{32}=m_{31}^{2}-q_{31}^{2}-2 m_{30} m_{32}, \quad B_{34}=m_{32}^{2}-2 m_{31} .
\end{aligned}
$$

From Eq. (2.18), we get

$$
\omega^{12}+e_{35} \omega^{10}+e_{34} \omega^{8}+e_{33} \omega^{6}+e_{32} \omega^{4}+e_{31} \omega^{2}+e_{30}=0
$$

where

$$
\begin{aligned}
& e_{30}=B_{30}^{2}-A_{30}^{2}, \quad e_{31}=2 B_{30} B_{32}-A_{31}^{2}-2 A_{30} A_{32}, \\
& e_{32}=B_{32}^{2}+2 B_{30} B_{34}-A_{32}^{2}-2 A_{30} A_{34}-2 A_{31} A_{33}, \\
& e_{33}=2 B_{30}+2 B_{32} B_{34}-A_{33}^{2}-2 A_{31} A_{35}-2 A_{32} A_{34}, \\
& e_{34}=B_{34}^{2}+2 B_{32}-A_{34}^{2}-2 A_{33} A_{35}, \quad e_{35}=2 B_{34}-A_{35}^{2} .
\end{aligned}
$$

Let $\omega^{2}=v_{3}$, then Eq. (2.19) can be written as

$$
v_{3}^{6}+e_{35} v_{3}^{5}+e_{34} v_{3}^{4}+e_{33} v_{3}^{3}+e_{32} v_{3}^{2}+e_{31} v_{3}+e_{30}=0 .
$$

Suppose that Eq. (2.20) has at least one positive root, and without loss of generality, we assume that it has six positive roots which are denoted by $v_{31}, v_{32}, v_{33}, v_{34}, v_{35}, v_{36}$, then Eq. (2.19) has six positive roots $\omega_{k}=\sqrt{v_{3 k}}, k=1,2,3,4,5,6$. The corresponding critical 
value of time delay $\tau_{k}^{(j)}$ is

$$
\begin{aligned}
\tau_{k}^{(j)} & =\frac{1}{\omega_{k}} \arccos \left\{\frac{A_{34} \omega_{k}^{4}+A_{32} \omega_{k}^{2}+A_{30}}{\omega_{k}^{6}+B_{34} \omega_{k}^{4}+B_{32} \omega_{k}^{2}+B_{30}}\right\}+\frac{2 \pi j}{\omega_{k}}, \\
k & =1,2,3,4,5,6, j=0,1,2, \ldots
\end{aligned}
$$

Then $\pm \omega_{k}$ is a pair of purely imaginary roots of Eq. (2.16) with $\tau=\tau_{k}^{(j)}$, and let $\tau_{0}=$ $\min _{k \in\{1-6\}}\left\{\tau_{k}^{(0)}\right\}, \omega_{0}=\omega_{k_{0}}$.

Lemma 2.4 Suppose that

$$
\text { (H31) } \quad A C+B D \neq 0
$$

holds, then the following transversality condition is satisfied:

$$
\left\{\frac{d(\operatorname{Re} \lambda)}{d \tau}\right\}_{\lambda=i \omega_{0}} \neq 0
$$

Proof Differentiating Eq. (2.16) with respect to $\tau$, we obtain

$$
\left(\frac{d \lambda}{d \tau}\right)^{-1}=\frac{2 n_{32} \lambda+n_{31}+\left(3 \lambda^{2}+2 m_{32} \lambda+m_{31}\right) e^{\lambda \tau}}{-\lambda\left(\lambda^{3}+m_{32} \lambda^{2}+m_{31} \lambda+m_{30}\right) e^{\lambda \tau}+\left(q_{31} \lambda^{2}+q_{30}\right) \lambda e^{-\lambda \tau}}-\frac{\tau}{\lambda},
$$

substituting $\lambda=i \omega_{0}$ into Eq. (2.22), we get

$$
\operatorname{Re}\left(\frac{d \lambda}{d \tau}\right)_{\lambda=i \omega_{0}}^{-1}=\operatorname{Re}\left(\frac{A+B i}{C+D i}\right)=\frac{A C+B D}{C^{2}+D^{2}}
$$

where

$$
\begin{aligned}
& A=\left(m_{31}-3 \omega_{0}^{2}\right) \cos \omega_{0} \tau_{0}-2 m_{32} \omega_{0} \sin \omega_{0} \tau_{0}+q_{31} \cos \omega_{0} \tau_{0}+n_{31}, \\
& B=\left(m_{31}-3 \omega_{0}^{2}\right) \sin \omega_{0} \tau_{0}+2 m_{32} \omega_{0} \cos \omega_{0} \tau_{0}-q_{31} \sin \omega_{0} \tau_{0}+2 n_{32} \omega_{0}, \\
& C=\left(m_{31}-q_{31}-\omega_{0}^{2}\right) \omega_{0}^{2} \cos \omega_{0} \tau_{0}+\left(q_{30}+m_{30}-m_{32} \omega_{0}^{2}\right) \omega_{0} \sin \omega_{0} \tau_{0}, \\
& D=\left(m_{31}+q_{31}-\omega_{0}^{2}\right) \omega_{0}^{2} \sin \omega_{0} \tau_{0}+\left(q_{30}-m_{30}+m_{32} \omega_{0}^{2}\right) \omega_{0} \cos \omega_{0} \tau_{0} .
\end{aligned}
$$

Noting that $\left\{\frac{d(\operatorname{Re} \lambda)}{d \tau}\right\}_{\lambda=i \omega_{0}}$ and $\left\{\operatorname{Re}\left(\frac{d \lambda}{d \tau}\right)^{-1}\right\}_{\lambda=i \omega_{0}}$ have the same sign, if condition (H31) holds, we obtain $\left\{\frac{d(\operatorname{Re} \lambda)}{d \tau}\right\}_{\lambda=i \omega_{0}} \neq 0$.

This completes the proof.

By applying Lemma 2.4 to Eq. (2.16), we obtain the existence of a Hopf bifurcation as stated in the following theorem.

Theorem 2.2 For system (1.2), $\tau_{1}=\tau_{2}=\tau \neq 0$. Suppose that condition $(\mathrm{H} 31)$ holds, then the positive equilibrium $E^{*}\left(x_{1}^{*}, x_{2}^{*}, y^{*}\right)$ is asymptotically stable for all $\tau \in\left[0, \tau_{0}\right)$ and unstable for $\tau>\tau_{0}$. Furthermore, system (1.2) undergoes a Hopf bifurcation at the positive equilibrium $E^{*}\left(x_{1}^{*}, x_{2}^{*}, y^{*}\right)$ when $\tau=\tau_{0}$. 
Case 4: $\tau_{1}>0, \tau_{2} \in\left[0, \tau_{20}\right)$, and $\tau_{1} \neq \tau_{2}$.

We consider Eq. (2.3) with $\tau_{2}$ in its stable interval, and $\tau_{1}$ is regarded as the parameter. Let $i \omega_{1_{*}}\left(\omega_{1_{*}}>0\right)$ be the root of Eq. (2.3), then we obtain

$$
\begin{aligned}
& E_{41} \sin \omega_{1_{*}} \tau_{1}+E_{42} \cos \omega_{1_{*}} \tau_{1}=E_{43}, \\
& E_{41} \cos \omega_{1_{*}} \tau_{1}-E_{42} \sin \omega_{1_{*}} \tau_{1}=E_{44},
\end{aligned}
$$

where

$$
\begin{aligned}
& E_{41}=n_{1} \omega_{1_{*}}-q_{0} \sin \omega_{1_{*}} \tau_{2}+q_{1} \omega_{1_{*}} \cos \omega_{1_{*}} \tau_{2}, \\
& E_{42}=n_{0}-n_{2} \omega_{1_{*}}^{2}+q_{0} \cos \omega_{1_{*}} \tau_{2}+q_{1} \omega_{1_{*}} \sin \omega_{1_{*}} \tau, \\
& E_{43}=m_{2} \omega_{1_{*}}^{2}-m_{0}+\left(p_{2} \omega_{1_{*}}^{2}-p_{0}\right) \cos \omega_{1_{*}} \tau_{2}-p_{1} \omega_{1_{*}} \sin \omega_{1_{*}} \tau_{2}, \\
& E_{44}=\omega_{1_{*}}^{3}-m_{1} \omega_{1_{*}}-\left(p_{2} \omega_{1_{*}}^{2}-p_{0}\right) \sin \omega_{1_{*}} \tau_{2}-p_{1} \omega_{1_{*}} \cos \omega_{1_{*}} \tau_{2} .
\end{aligned}
$$

From Eq. (2.24), we have

$$
\begin{aligned}
& \omega_{1_{*}}^{6}+e_{42} \omega_{1_{*}}^{4}+e_{41} \omega_{1_{*}}^{2}+e_{40}+\left(c_{44} \omega_{1_{*}}^{4}+c_{42} \omega_{1_{*}}^{2}+c_{40}\right) \cos \omega_{1_{*}} \tau_{2} \\
& +\left(c_{45} \omega_{1_{*}}^{5}+c_{43} \omega_{1_{*}}^{3}+c_{41} \omega_{1_{*}}\right) \sin \omega_{1_{*}} \tau_{2}=0
\end{aligned}
$$

where

$$
\begin{aligned}
& e_{40}=m_{0}^{2}+p_{0}^{2}-n_{0}^{2}-q_{0}^{2}, \quad e_{41}=m_{1}^{2}+p_{1}^{2}-n_{1}^{2}-q_{1}^{2}+2 n_{0} n_{2}-2 m_{0} m_{2}-2 p_{0} p_{2}, \\
& e_{42}=m_{2}^{2}-n_{2}^{2}-2 m_{1}+p_{2}^{2}, \quad c_{40}=2 m_{0} p_{0}-2 n_{0} q_{0}, \\
& c_{41}=2 p_{1} m_{0}-2 p_{0} m_{1}+2 n_{1} q_{0}-2 n_{0} q_{1}, \\
& c_{42}=2 p_{1} m_{1}-2 p_{0} m_{2}+2 n_{2} q_{0}-2 p_{2} m_{0}-2 n_{1} q_{1}, \\
& c_{43}=2 p_{0}-2 p_{1} m_{2}+2 p_{2} m_{1}+2 n_{2} q_{1}, \quad c_{44}=-2 p_{1}+2 p_{2} m_{2}, \quad c_{45}=-2 p_{2} .
\end{aligned}
$$

In order to give the main results, we provide the following assumption.

(H41) Eq. (2.25) has at least a finite positive root.

We denote the positive roots of Eq. (2.25) by $\omega_{1_{*}}^{(1)}, \omega_{1_{*}}^{(2)}, \omega_{1_{*}}^{(3)}, \omega_{1_{*}}^{(4)}, \omega_{1_{*}}^{(5)}$, and $\omega_{1_{*}}^{(6)}$. For every $\omega_{1_{*}}^{(i)}(i=1,2,3,4,5,6)$, the corresponding critical value of time delay $\tau_{1 i}^{(j)}, j=1,2,3 \ldots$, is

$$
\begin{aligned}
\tau_{1 i}^{(j)} & =\frac{1}{\omega_{1_{*}}} \arccos \left\{\frac{E_{41} E_{44}+E_{42} E_{43}}{E_{41}^{2}+E_{42}^{2}}+2 \pi j\right\}_{\omega_{1_{*}}=\omega_{1_{*}}^{i}}, \\
i & =1,2,3,4,5,6 ; j=0,1,2 \ldots
\end{aligned}
$$

Let $\tau_{10}^{\prime}=\min \left\{\tau_{1 i}^{(0)} \mid i=1,2, \ldots 6 ; j=0,1,2 \ldots\right\}, \omega_{10}^{\prime}$ is the corresponding root of Eq. (2.25) with $\tau_{10}^{\prime}$.

\section{Lemma 2.5 Suppose that}

(H42) $\quad A^{\prime} C^{\prime}+B^{\prime} D^{\prime} \neq 0$ 
holds, then the following transversality condition holds:

$$
\left\{\frac{d(\operatorname{Re} \lambda)}{d \tau_{1}}\right\}_{\lambda=i \omega_{10}^{\prime}} \neq 0
$$

Proof Taking the derivative of $\lambda$ with respect to $\tau_{1}$ in Eq. (2.3) and substituting $\lambda=i \omega_{10}^{\prime}$, we get

$$
\operatorname{Re}\left(\frac{d \lambda}{d \tau_{1}}\right)_{\lambda=i \omega_{10}}^{\prime-1}=\operatorname{Re}\left(\frac{A^{\prime}+B^{\prime} i}{C^{\prime}+D^{\prime} i}\right)=\frac{A^{\prime} C^{\prime}+B^{\prime} D^{\prime}}{C^{\prime 2}+D^{\prime 2}}
$$

where

$$
\begin{aligned}
A^{\prime}= & m_{1}-3 \omega_{10}^{\prime 2}+2 n_{2} \omega_{10}^{\prime} \sin \omega_{10}^{\prime} \tau_{10}^{\prime}+n_{1} \cos \omega_{10}^{\prime} \tau_{10}^{\prime} \\
& +\sin \omega_{10}^{\prime} \tau_{2}\left(-p_{1} \omega_{10}^{\prime} \tau_{2}+2 p_{2} \omega_{10}^{\prime}+q_{1} \sin \omega_{10}^{\prime} \tau_{10}^{\prime}\right) \\
& +\cos \omega_{10}^{\prime} \tau_{2}\left(p_{2} \tau_{2} \omega_{10}^{\prime 2}+p_{1}-p_{0} \tau_{2}+q_{1} \cos \omega_{10}^{\prime} \tau_{10}^{\prime}\right), \\
B^{\prime}= & 2 m_{2} \omega_{10}^{\prime}-n_{1} \sin \omega_{10}^{\prime} \tau_{10}^{\prime}+2 n_{2} \omega_{10}^{\prime} \cos \omega_{10}^{\prime} \tau_{10}^{\prime} \\
& +\sin \omega_{10}^{\prime} \tau_{2}\left(-p_{1}+p_{0} \tau_{2}-p_{2} \tau_{2} \omega_{10}^{\prime 2}-q_{1} \cos \omega_{10}^{\prime} \tau_{10}^{\prime}\right) \\
& +\cos \omega_{10}^{\prime} \tau_{2}\left(2 p_{2} \omega_{10}^{\prime}-p_{1} \omega_{10}^{\prime} \tau_{2}-q_{1} \sin \omega_{10}^{\prime} \tau_{10}^{\prime}\right), \\
C^{\prime}= & \left(n_{0} \omega_{10}^{\prime}-n_{2} \omega_{10}^{\prime 3}\right) \sin \omega_{10}^{\prime} \tau_{10}^{\prime}-n_{1} \omega_{10}^{\prime 2} \cos \omega_{10}^{\prime} \tau_{10}^{\prime} \\
& +\left(q_{0} \omega_{10}^{\prime} \cos \omega_{10}^{\prime} \tau_{10}^{\prime}+q_{1} \omega_{10}^{\prime 2} \sin \omega_{10}^{\prime} \tau_{10}^{\prime}\right) \sin \omega_{10}^{\prime} \tau_{2} \\
& +\left(q_{0} \omega_{10}^{\prime} \sin \omega_{10}^{\prime} \tau_{10}^{\prime}-q_{1} \omega_{10}^{\prime 2} \cos \omega_{10}^{\prime} \tau_{10}^{\prime}\right) \cos \omega_{10}^{\prime} \tau_{2}, \\
D^{\prime}= & \left(n_{0} \omega_{10}^{\prime}-n_{2} \omega_{10}^{\prime 3}\right) \cos \omega_{10}^{\prime} \tau_{10}^{\prime}+n_{1} \omega_{10}^{\prime 2} \sin \omega_{10}^{\prime} \tau_{10}^{\prime} \\
& +\left(-q_{0} \omega_{10}^{\prime} \sin \omega_{10}^{\prime} \tau_{10}^{\prime}+q_{1} \omega_{10}^{\prime 2} \cos \omega_{10}^{\prime} \tau_{10}^{\prime}\right) \sin \omega_{10}^{\prime} \tau_{2} \\
& +\left(q_{0} \omega_{10}^{\prime} \cos \omega_{10}^{\prime} \tau_{10}^{\prime}+q_{1} \omega_{10}^{\prime 2} \sin \omega_{10}^{\prime} \tau_{10}^{\prime}\right) \cos \omega_{10}^{\prime} \tau_{2} .
\end{aligned}
$$

Obviously, if condition (H42) holds, then we have $\left\{\frac{d(\operatorname{Re} \lambda)}{d \tau_{1}}\right\}_{\lambda=i \omega_{10}^{\prime}} \neq 0$. This completes the proof of the lemma.

By the above analysis, we have the following theorem.

Theorem 2.3 For system (1.2), $\tau_{1}>0, \tau_{2} \in\left[0, \tau_{20}\right)$, and $\tau_{1} \neq \tau_{2}$. Suppose that conditions $(\mathrm{H} 41)$ and $(\mathrm{H} 42)$ hold, then the positive equilibrium $E^{*}\left(x_{1}^{*}, x_{2}^{*}, y^{*}\right)$ is asymptotically stable for all $\tau_{1} \in\left[0, \tau_{10}^{\prime}\right)$ and unstable for $\tau_{1}>\tau_{10}^{\prime}$. Furthermore, system (1.2) undergoes a Hopf bifurcation at the positive equilibrium $E^{*}\left(x_{1}^{*}, x_{2}^{*}, y^{*}\right)$ when $\tau_{1}=\tau_{10}^{\prime}$.

\section{Direction and stability of Hopf bifurcation}

In the previous section, we have shown that system (1.2) undergoes a Hopf bifurcation for different combinations of $\tau_{1}$ and $\tau_{2}$. In this section, we shall study the direction of Hopf bifurcation and the stability of bifurcating periodic solutions of system (1.2) with respect to $\tau_{1}$ and $\tau_{2} \in\left[0, \tau_{20}\right)$. The theoretical approach we apply is based on the normal form theory and center manifold theorem [18]. Throughout this section, we assume that system (1.2) undergoes a Hopf bifurcation at $\tau_{1}=\tau_{10}^{\prime}, \tau_{2} \in\left[0, \tau_{20}\right)$. 
Without loss of generality, we assume that $\tau_{10}^{\prime}>\tau_{2}^{\prime}$. Let $\tau_{1}=\tau_{10}^{\prime}+\mu, \mu \in R, t=s \tau_{1}$, $x_{1}\left(s \tau_{1}\right)=\hat{x}_{1}(s), x_{2}\left(s \tau_{1}\right)=\hat{x}_{2}(s), y\left(s \tau_{1}\right)=\hat{y}(s)$. Denote $x_{1}=\hat{x}_{1}, x_{2}=\hat{x}_{2}, y=\hat{y}$, and $t=s$, then system (1.2) can be written as a functional differential equation (FDE) in $C=C\left([-1,0], R^{3}\right)$ :

$$
u^{\prime}(t)=L_{\mu}\left(u_{t}\right)+F\left(\mu, u_{t}\right)
$$

where $u(t)=\left(x_{1}(t), x_{2}(t), y(t)\right)^{T} \in C$, and

$$
\begin{aligned}
& u_{t}(\theta)=u(t+\theta)=\left(x_{1}(t+\theta), x_{2}(t+\theta), y(t+\theta)\right)^{T} \in C, \quad \text { and } \\
& L_{\mu}: C \rightarrow R^{3}, \quad F: R \times C \rightarrow R^{3}
\end{aligned}
$$

are given by

$$
L_{\mu}(\phi)=\left(\tau_{10}^{\prime}+\mu\right) \tilde{A} \phi(0)+\left(\tau_{10}^{\prime}+\mu\right) \tilde{B} \phi\left(-\frac{\tau_{2}^{\prime}}{\tau_{10}^{\prime}}\right)+\left(\tau_{10}^{\prime}+\mu\right) \tilde{C} \phi(-1),
$$

and

$$
F(\mu, \phi)=\left(\tau_{10}^{\prime}+\mu\right)\left(F_{1}, F_{2}, F_{3}\right)^{T},
$$

where

$$
\begin{aligned}
\phi(\theta)= & \left(\phi_{1}(\theta), \phi_{2}(\theta), \phi_{3}(\theta)\right)^{T} \in C, \\
\tilde{A}= & \left(\begin{array}{ccc}
a_{11} & a_{12} & a_{13} \\
a_{21} & a_{22} & 0 \\
0 & 0 & a_{33}
\end{array}\right), \quad \tilde{B}=\left(\begin{array}{ccc}
0 & 0 & 0 \\
0 & 0 & 0 \\
b_{31} & 0 & b_{33}
\end{array}\right), \quad \tilde{C}=\left(\begin{array}{ccc}
b_{11} & 0 & 0 \\
0 & 0 & 0 \\
0 & 0 & 0
\end{array}\right), \\
F_{1}= & k_{11} \phi_{1}^{2}(0)+k_{12} \phi_{1}(0) \phi_{1}(-1)+k_{13} \phi_{1}(0) \phi_{3}(0)+k_{14} \phi_{1}^{2}(0) \phi_{3}(0)+k_{15} \phi_{1}^{3}(0)+\cdots, \\
F_{2}= & 0, \\
F_{3}= & k_{31} \phi_{1}^{2}\left(-\frac{\tau_{2}^{\prime}}{\tau_{10}^{\prime}}\right)+k_{32} \phi_{1}\left(-\frac{\tau_{2}^{\prime}}{\tau_{10}^{\prime}}\right) \phi_{3}\left(-\frac{\tau_{2}^{\prime}}{\tau_{10}^{\prime}}\right) \\
& +k_{33} \phi_{1}^{2}\left(-\frac{\tau_{2}^{\prime}}{\tau_{10}^{\prime}}\right) \phi_{3}\left(-\frac{\tau_{2}^{\prime}}{\tau_{10}^{\prime}}\right)+k_{34} \phi_{1}^{3}\left(-\frac{\tau_{2}^{\prime}}{\tau_{10}^{\prime}}\right)+\cdots, \\
k_{11}= & \frac{-2 a_{1} y^{*}+6 a_{1} m\left(x_{1}^{*}\right)^{2} y^{*}}{\left(1+m\left(x_{1}^{*}\right)^{2}\right)^{3}}, \quad k_{12}=-c, \\
k_{14}= & \frac{-2 a_{1}+6 a_{1} m\left(x_{1}^{*}\right)^{2}}{\left(1+m\left(x_{1}^{*}\right)^{2}\right)^{3}}, \quad k_{15}=\frac{24 a_{1} m x_{1}^{*} y^{*}\left(1-m\left(x_{1}^{*}\right)^{2}\right)}{\left(1+m\left(x_{1}^{*}\right)^{2}\right)^{4}}, \\
k_{31}= & \frac{2 a_{2} y^{*}-6 a_{2} m\left(x_{1}^{*}\right)^{2} y^{*}}{\left(1+m\left(x_{1}^{*}\right)^{2}\right)^{3}}, \quad k_{32}=\frac{2 a_{2} x_{1}^{*}}{\left(1+m\left(x_{1}^{*}\right)^{2}\right)^{2}}, \\
k_{33}= & \frac{2 a_{2}-6 a_{2} m\left(x_{1}^{*}\right)^{2}}{\left(1+m\left(x_{1}^{*}\right)^{2}\right)^{3}}, \quad k_{34}=\frac{24 a_{2} m x_{1}^{*} y^{*}\left(-1+m\left(x_{1}^{*}\right)^{2}\right)}{\left(1+m\left(x_{1}^{*}\right)^{2}\right)^{4}} .
\end{aligned}
$$

Hence, by the Riesz representation theorem, there exists a $3 \times 3$ matrix function $\eta(\theta, \mu)$ of bounded variation for $\theta \in[-1,0]$ such that

$$
L_{\mu} \phi=\int_{-1}^{0} d \eta(\theta, \mu) \phi(\theta) \quad \text { for } \phi \in C .
$$


In fact, we can choose

$$
\eta(\theta, \mu)= \begin{cases}\left(\tau_{10}^{\prime}+\mu\right)(\tilde{A}+\tilde{B}+\tilde{C}), & \theta=0, \\ \left(\tau_{10}^{\prime}+\mu\right)(\tilde{B}+\tilde{C}), & \theta \in\left[-\frac{\tau_{2}^{\prime}}{\tau_{10}}, 0\right), \\ \left(\tau_{10}+\mu\right) \tilde{C}, & \theta \in\left(-1,-\frac{\tau_{2}^{\prime}}{\tau_{10}}\right), \\ 0, & \theta=-1 .\end{cases}
$$

For $\phi \in C\left([-1,0], R^{3}\right)$, define

$$
A(\mu) \phi= \begin{cases}\frac{d \phi(\theta)}{d \theta}, & -1 \leq \theta<0 \\ \int_{-1}^{0} d \eta(\theta, \mu) \phi(\theta), & \theta=0\end{cases}
$$

and

$$
R_{\mu}(\phi)= \begin{cases}0, & -1 \leq \theta<0 \\ F(\mu, \phi), & \theta=0\end{cases}
$$

Then Eq. (3.1) can be transformed into the following operator equation:

$$
u_{t}^{\prime}=A(\mu) u_{t}+R(\mu) u_{t}
$$

For $\varphi \in C^{\prime}\left([-1,0],\left(R^{3}\right)^{*}\right)$, where $\left(R^{3}\right)^{*}$ is the three-dimensional space of row vectors, we further define the adjoint operator $A^{*}$ of $A(0)$ :

$$
A^{*} \varphi(s)= \begin{cases}-\frac{d \varphi(s)}{d s}, & s \in(0,1] \\ \int_{-1}^{0} d \eta^{T}(t, 0) \varphi(-t), & s=0\end{cases}
$$

For $\phi \in C\left([-1,0], R^{3}\right)$ and $\varphi \in C^{\prime}\left([-1,0],\left(R^{3}\right)^{*}\right)$, define the bilinear form

$$
\langle\varphi(s), \phi(s)\rangle=\bar{\varphi}(0) \phi(0)-\int_{-1}^{0} \int_{\xi=0}^{\theta} \bar{\varphi}(\xi-\theta) d \eta(\theta) \phi(\xi) d \xi
$$

where $\eta(\theta)=\eta(\theta, 0), A=A(0)$ and $A^{*}$ are adjoint operators. From Sect. 2, we know that $\pm i \omega_{10}^{\prime} \tau_{10}^{\prime}$ are eigenvalues of $A(0)$. Thus they are also the eigenvalues of $A^{*}$.

Suppose that $q(\theta)=\left(1, q_{2}, q_{3}\right)^{T} e^{i \omega_{10}^{\prime} \tau_{10}^{\prime} \theta}$ is the eigenvector of $A(0)$ corresponding to $i \omega_{10}^{\prime} \tau_{10}^{\prime}$ and $q^{*}(s)=1 / \rho\left(1, q_{2}^{*}, q_{3}^{*}\right) e^{i \omega_{10}^{\prime} \tau_{10}^{\prime} s}$ is the eigenvector of $A^{*}$ corresponding to $-i \omega_{10}^{\prime} \tau_{10}^{\prime}$. By the direct calculation, we obtain

$$
\begin{array}{ll}
q_{2}=\frac{a_{21}}{i \omega_{10}^{\prime}-a_{22}}, & q_{3}=\frac{b_{31} e^{-i \omega_{10}^{\prime} \tau_{2}^{\prime}}}{i \omega_{10}^{\prime}-a_{33}-b_{33} e^{-i \omega_{10}^{\prime} \tau_{2}^{\prime}}}, \\
q_{2}^{*}=-\frac{a_{12}}{i \omega_{10}^{\prime}+a_{22}}, & q_{3}^{*}=-\frac{a_{13}}{i \omega_{10}^{\prime}+a_{33}+b_{33} e^{-i \omega_{10}^{\prime} \tau_{2}^{\prime}}} .
\end{array}
$$


Then, from Eq. (3.10), we get

$$
\begin{aligned}
\left\langle q^{*}(s), q(\theta)\right\rangle & =\bar{q}^{*}(0) q(0)-\int_{-1}^{0} \int_{\xi=0}^{\theta} \bar{q}^{*}(\xi-\theta) d \eta(\theta) q(\xi) d \xi \\
& =\frac{1}{\bar{\rho}}\left[1+q_{2} \bar{q}_{2}^{*}+q_{3} \bar{q}_{3}^{*}-\int_{-1}^{0}\left(1, \bar{q}_{2}^{*}, \bar{q}_{3}^{*}\right) \theta e^{i \omega_{10}^{\prime} \tau_{10}^{\prime} \theta} d \eta(\theta)\left(1, q_{2}, q_{3}\right)^{T}\right] \\
& =\frac{1}{\bar{\rho}}\left[1+q_{2} \bar{q}_{2}^{*}+q_{3} \bar{q}_{3}^{*}+b_{11} \tau_{10}^{\prime} e^{-i \omega_{10}^{\prime} \tau_{10}^{\prime}}+\tau_{2}^{\prime} e^{-i \omega_{10}^{\prime} \tau_{2}^{\prime}} \bar{q}_{3}^{*}\left(b_{31}+b_{33} q_{3}\right)\right] .
\end{aligned}
$$

Therefore, we can choose

$$
\bar{\rho}=1+q_{2} \bar{q}_{2}^{*}+q_{3} \bar{q}_{3}^{*}+b_{11} \tau_{10}^{\prime} e^{-i \omega_{10}^{\prime} \tau_{10}^{\prime}}+\tau_{2}^{\prime} e^{-i \omega_{10}^{\prime} \tau_{2}^{\prime}} \bar{q}_{3}^{*}\left(b_{31}+b_{33} q_{3}\right)
$$

such that $\left\langle q^{*}(s), q(\theta)\right\rangle=1,\left\langle q^{*}(s), \bar{q}(\theta)\right\rangle=0$.

In the remainder of this section, by using the algorithms in [18] and using a similar calculation process to that in [10], we obtain the coefficients used in determining the direction of Hopf bifurcation and the stability of the bifurcation periodic solutions:

$$
\begin{aligned}
g_{20}= & \frac{2 \tau_{10}^{\prime}}{\bar{\rho}}\left[k_{11}+k_{12} e^{-i \omega_{10}^{\prime} \tau_{10}^{\prime}}+k_{13} q_{3}+\bar{q}_{3}^{*}\left(k_{31} e^{-2 i \omega_{10}^{\prime} \tau_{2}^{\prime}}+k_{32} q_{3} e^{-2 i \omega_{10}^{\prime} \tau_{2}^{\prime}}\right)\right] \\
g_{11}= & \frac{\tau_{10}^{\prime}}{\bar{\rho}}\left[2 k_{11}+k_{12}\left(e^{-i \omega_{10}^{\prime} \tau_{10}^{\prime}}+e^{i \omega_{10}^{\prime} \tau_{10}^{\prime}}\right)+k_{13}\left(q_{3}+\bar{q}_{3}\right)+\bar{q}_{3}^{*}\left(2 k_{31}+k_{32}\left(q_{3}+\bar{q}_{3}\right)\right]\right. \\
g_{02}= & \frac{2 \tau_{10}^{\prime}}{\bar{\rho}}\left[k_{11}+k_{12} e^{i \omega_{10}^{\prime} \tau_{10}^{\prime}}+k_{13} \bar{q}_{3}+\bar{q}_{3}^{*}\left(k_{31} e^{2 i \omega_{10}^{\prime} \tau_{2}^{\prime}}+k_{32} \bar{q}_{3} e^{2 i \omega_{10}^{\prime} \tau_{2}^{\prime}}\right)\right] \\
g_{21}= & \frac{2 \tau_{10}^{\prime}}{\bar{\rho}}\left[k_{11}\left(W_{20}^{(1)}(0)+2 W_{11}^{(1)}(0)\right)+k_{12}\left(W_{11}^{(1)}(-1)+\frac{1}{2} W_{20}^{(1)}(-1)\right.\right. \\
& \left.+\frac{1}{2} W_{20}^{(1)}(0) e^{i \omega_{10}^{\prime} \tau_{10}^{\prime}}+W_{11}^{(1)}(0) e^{-i \omega_{10}^{\prime} \tau_{10}^{\prime}}\right) \\
+ & k_{13}\left(W_{11}^{(3)}(0)+\frac{1}{2} W_{20}^{(3)}(0)+\frac{1}{2} W_{20}^{(1)}(0) \bar{q}_{3}+W_{11}^{(1)}(0) q_{3}\right)+k_{14}\left(2 q_{3}+\bar{q}_{3}\right) \\
& +\bar{q}_{3}^{*}\left[k_{31}\left(2 W_{11}^{(1)}\left(-\frac{\tau_{2}^{\prime}}{\tau_{10}^{\prime}}\right) e^{-i \omega_{10}^{\prime} \tau_{2}^{\prime}}+W_{20}^{(1)}\left(-\frac{\tau_{2}^{\prime}}{\tau_{10}^{\prime}}\right) \bar{q}_{3} e^{i \omega_{10}^{\prime} \tau_{2}^{\prime}}\right)\right. \\
& +k_{32}\left(W_{11}^{(3)}\left(-\frac{\tau_{2}^{\prime}}{\tau_{10}^{\prime}}\right) e^{-i \omega_{10}^{\prime} \tau_{2}^{\prime}}+\frac{1}{2} W_{20}^{(3)}\left(-\frac{\tau_{2}^{\prime}}{\tau_{10}^{\prime}}\right) e^{i \omega_{10}^{\prime} \tau_{2}^{\prime}}\right. \\
& \left.+\frac{1}{2} \bar{q}_{3} W_{20}^{(1)}\left(-\frac{\tau_{2}^{\prime}}{\tau_{10}^{\prime}}\right) e^{i \omega_{10}^{\prime} \tau_{2}^{\prime}}+q_{3} W_{11}^{(1)}\left(-\frac{\tau_{2}^{\prime}}{\tau_{10}^{\prime}}\right) e^{-i \omega_{10}^{\prime} \tau_{2}^{\prime}}\right) \\
& \left.\left.+k_{33}\left(2 q_{3} e^{-i \omega_{10}^{\prime} \tau_{2}^{\prime}}+\bar{q}_{3} e^{-i \omega_{10}^{\prime} \tau_{2}}\right)+k_{34}\left(3 e^{-i \omega_{10}^{\prime} \tau_{2}^{\prime}}\right)\right]\right]
\end{aligned}
$$

However,

$$
\begin{aligned}
& W_{20}(\theta)=\frac{i g_{20}}{\omega_{10}^{\prime} \tau_{10}^{\prime}} q(0) e^{i \omega_{10}^{\prime} \tau_{10}^{\prime} \theta}+\frac{i \bar{g}_{02}}{3 \omega_{10}^{\prime} \tau_{10}^{\prime}} \bar{q}(0) e^{-i \omega_{10}^{\prime} \tau_{10}^{\prime} \theta}+E_{1} e^{2 i \omega_{10}^{\prime} \tau_{10}^{\prime} \theta}, \\
& W_{11}(\theta)=-\frac{i g_{11}}{\omega_{10}^{\prime} \tau_{10}^{\prime}} q(0) e^{i \omega_{10}^{\prime} \tau_{10}^{\prime} \theta}+\frac{i \bar{g}_{11}}{\omega_{10}^{\prime} \tau_{10}^{\prime}} \bar{q}(0) e^{-i \omega_{10}^{\prime} \tau_{10}^{\prime} \theta}+E_{2},
\end{aligned}
$$


where $E_{1}=\left(E_{1}^{(1)}, E_{1}^{(2)}, E_{1}^{(3)}\right)^{T} \in R^{3}$ and $E_{2}=\left(E_{2}^{(1)}, E_{2}^{(2)}, E_{2}^{(3)}\right)^{T} \in R^{3}$ are also constant vectors and can be determined by the following equations, respectively:

$$
\begin{aligned}
& \left(\begin{array}{ccc}
2 i \omega_{10}^{\prime}-a_{11}-b_{11} e^{-2 i \omega_{10}^{\prime} \tau_{10}^{\prime}} & -a_{12} & -a_{13} \\
0 & 2 i \omega_{10}^{\prime}-a_{22} & 0 \\
-b_{31} e^{-2 i \omega_{10}^{\prime} \tau_{2}^{\prime}} & 0 & 2 i \omega_{10}^{\prime}-a_{33}-b_{33} e^{-2 i \omega_{10}^{\prime} \tau_{2}^{\prime}}
\end{array}\right) E_{1} \\
& =2\left(\begin{array}{c}
H_{1} \\
H_{2} \\
H_{3}
\end{array}\right), \\
& \left(\begin{array}{ccc}
-a_{11}-b_{11} & -a_{12} & -a_{13} \\
0 & -a_{22} & 0 \\
-b_{31} & 0 & -a_{33}-b_{33}
\end{array}\right) E_{2}=\left(\begin{array}{l}
P_{1} \\
P_{2} \\
P_{3}
\end{array}\right)
\end{aligned}
$$

with

$$
\begin{aligned}
& H_{1}=k_{11}+k_{12} e^{-i \omega_{10}^{\prime} \tau_{10}^{\prime}}+k_{13} q_{3}, \\
& H_{2}=0, \\
& H_{3}=k_{31} e^{-2 i \omega_{10}^{\prime} \tau_{2}^{\prime}}+k_{32} q_{3} e^{-2 i \omega_{10}^{\prime} \tau_{2}^{\prime},} \\
& P_{1}=2 k_{11}+k_{12}\left(e^{-i \omega_{10}^{\prime} \tau_{10}^{\prime}}+e^{i \omega_{10}^{\prime} \tau_{10}^{\prime}}\right)+k_{13}\left(q_{3}+\bar{q}_{3}\right), \\
& P_{2}=0, \\
& P_{3}=2 k_{31}+k_{32}\left(q_{3}+\bar{q}_{3}\right) .
\end{aligned}
$$

Therefore, we can calculate $g_{21}$ and compute the following values:

$$
\begin{aligned}
& c_{1}(0)=\frac{i}{2 \omega_{10}^{\prime} \tau_{10}^{\prime}}\left(g_{20} g_{11}-2\left|g_{11}\right|^{2}-\frac{\left|g_{02}\right|^{2}}{3}\right)+\frac{g_{21}}{2}, \\
& \mu_{2}=-\frac{\operatorname{Re}\left\{c_{1}(0)\right\}}{\operatorname{Re}\left\{\lambda^{\prime}\left(\tau_{10}^{\prime}\right)\right\}} \\
& \beta_{2}=2 \operatorname{Re}\left(c_{1}(0)\right) \\
& T_{2}=-\frac{\operatorname{Im}\left\{c_{1}(0)\right\}+\mu_{2} \operatorname{Im}\left\{\lambda^{\prime}\left(\tau_{10}\right)\right\}}{\omega_{10}^{\prime} \tau_{10}^{\prime}},
\end{aligned}
$$

which determine the properties of bifurcating periodic solutions at $\tau=\tau_{10}^{\prime}$ on the center manifold. From the discussion above, we have the following result.

Theorem 3.1 For system (1.2), the direction of Hopf bifurcation is determined by the sign of $\mu_{2}$ : if $\mu_{2}>0\left(\mu_{2}<0\right)$, then the Hopfbifurcation is supercritical (subcritical). The stability of the bifurcating periodic solutions is determined by the sign of $\beta_{2}$ : if $\beta_{2}<0\left(\beta_{2}>0\right)$, the bifurcating periodic solutions are stable (unstable). The period of the bifurcating periodic solutions is determined by the sign of $T_{2}$ : if $T_{2}>0\left(T_{2}<0\right)$, the bifurcating periodic solutions increase (decrease). 


\section{Numerical examples}

In this section, we give some numerical simulations by using matlab to illustrate the analytical results in the previous section.

Let $a=8, b=1, c=1, a_{1}=3, r_{1}=0.5, a_{2}=2, r_{2}=r=1, m=1$. Then we have the following particular example of system (1.2):

$$
\begin{aligned}
& \dot{x}_{1}(t)=8 x_{2}(t)-0.5 x_{1}(t)-x_{1}(t)-x_{1}(t) x_{1}\left(t-\tau_{1}\right)-\frac{3 x_{1}^{2}(t) y(t)}{1+x_{1}^{2}(t)}, \\
& \dot{x}_{2}(t)=x_{1}(t)-x_{2}(t), \\
& \dot{y}(t)=\frac{2 x_{1}^{2}\left(t-\tau_{2}\right) y\left(t-\tau_{2}\right)}{1+x_{1}^{2}\left(t-\tau_{2}\right)}-y(t) .
\end{aligned}
$$

It is not difficult to verify that condition (H1) holds, we obtain the positive equilibrium $E^{*}\left(1,1, \frac{11}{3}\right)$.

For $\tau_{1}=0, \tau_{2}>0$, we obtain $w_{20} \approx 0.4609, \tau_{20} \approx 1$.9387. From Theorem 2.1 , we know that the positive equilibrium $E^{*}$ is asymptotically stable when $\tau_{2} \in\left[0, \tau_{20}\right)$, when the time delay $\tau_{2}$ passes through the critical value $\tau_{20}$, the interior equilibrium $E^{*}$ will lose its stability and a Hopf bifurcation occurs, and a family of periodic solutions bifurcate from the interior equilibrium $E^{*}$. The corresponding waveform and the phase plots are depicted in Fig. 1 and Fig. 2.

For $\tau_{1}=\tau_{2}=\tau$, we get $\omega_{0}=0.5385, \tau_{0}=1.7889$. By Theorem 2.2, we know that, when $\tau$ increases from zero to the critical value $\tau_{0}$, the equilibrium point $E^{*}$ is asymptotically stable. Once the time delay $\tau$ passes through the critical value $\tau_{0}$, the positive equilibrium

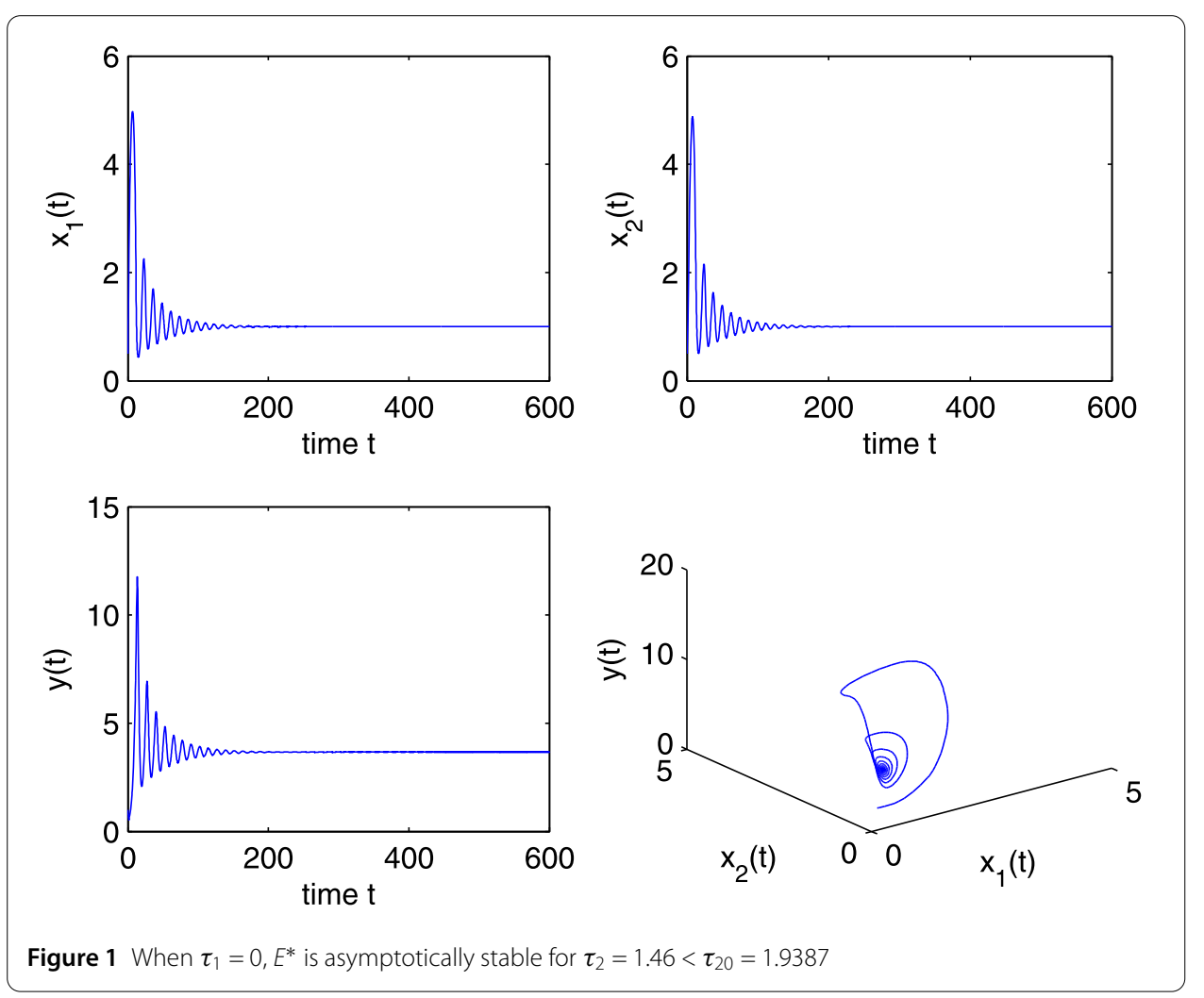



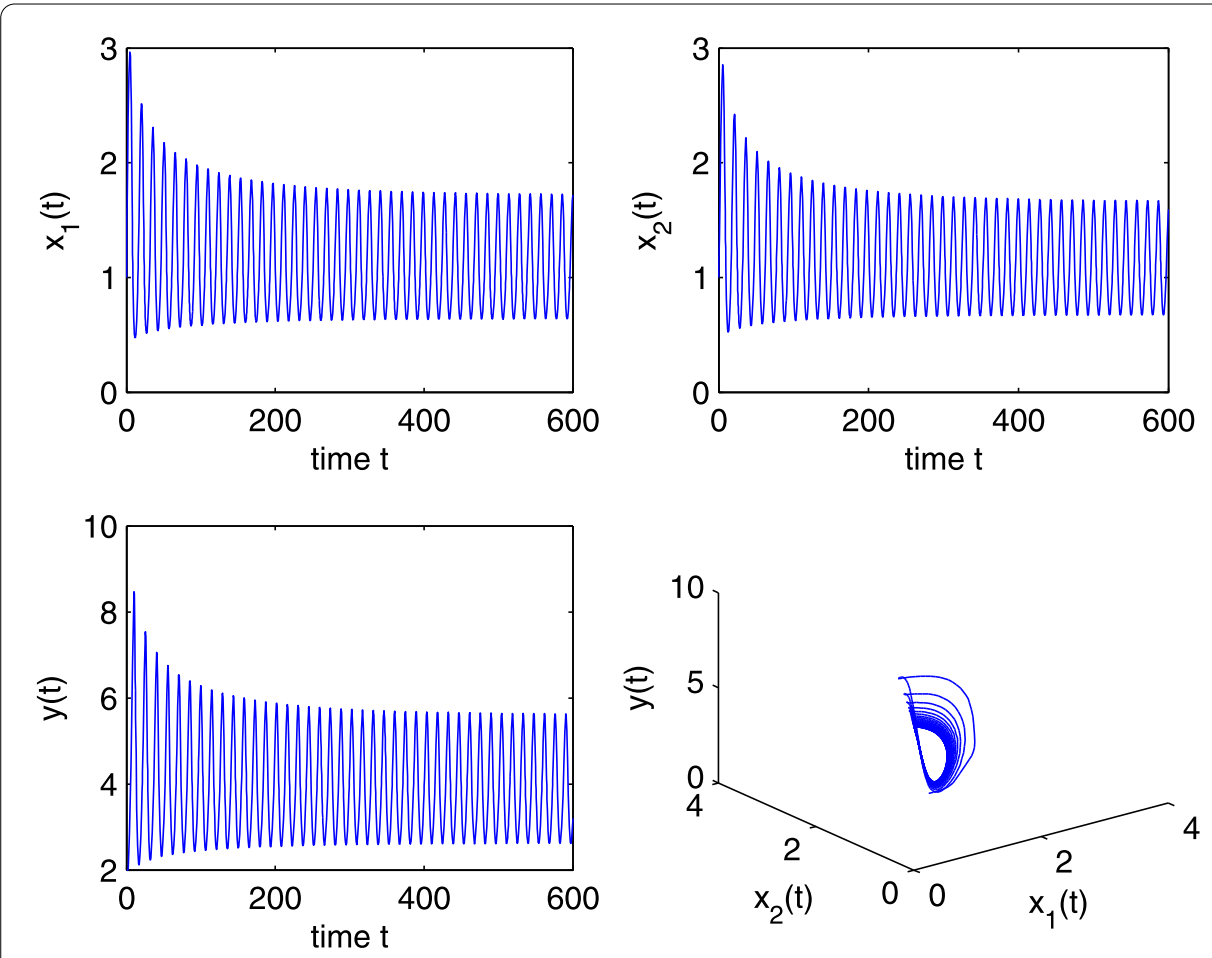

Figure 2 When $\tau_{1}=0, E^{*}$ undergoes a Hopf bifurcation for $\tau_{2}=1.98>\tau_{20}=1.9387$

$E^{*}$ will lose its stability and a Hopf bifurcation occurs, which can be depicted in Fig. 3 and Fig. 4.

For $\tau_{1}>0, \tau_{2}^{\prime}=1.5 \in\left[0, \tau_{20}\right)$, we have $\omega_{10}^{\prime}=0.5974, \tau_{10}^{\prime}=2.3522$. According to Theorem $2.3, E^{*}$ is asymptotically stable when $\tau_{1} \in\left[0, \tau_{10}^{\prime}\right)$ and unstable when $\tau_{1}>\tau_{10}^{\prime}$. After the computation of Eq. (3.17), we obtain $c_{1}(0)=-22.3543+6.0903 i, \mu_{2}=444.4195$, $\beta_{2}=-44.7086, T_{2}=-11.6715$. From Theorem 3.1, the Hopf bifurcation is supercritical, the bifurcating periodic solutions are stable, which is shown in Fig. 5 and Fig. 6.

Numerical simulations illustrate our theoretical analysis. Owing to the bifurcation periodic solutions being stable, the species in system (1.2) can coexist in an oscillatory mode from the viewpoint of biology.

\section{Conclusions}

In this paper, we have studied the problem of Hopf bifurcation analysis in a delayed predator-prey model with stage structure for the prey. By setting the same group of parameter values, according to the existing two time delays and discussing four different cases, we know that the interior equilibrium will lose its original stability and a Hopf bifurcation occurs, and a family of periodic solutions bifurcate $E^{*}$ when the time delay passes though some critical values. By using the normal form theory and center manifold theorem, the explicit formulas which determine the direction of Hopf bifurcation and the stability of the bifurcating periodic solution are derived. The numerical results that the Hopf bifurcation is supercritical and the bifurcation periodic solutions are stable are in excellent agreement with theoretical analysis, that is, the number of predators and prey implies stability and coexistence. Therefore, the research about this kind of model has cer- 

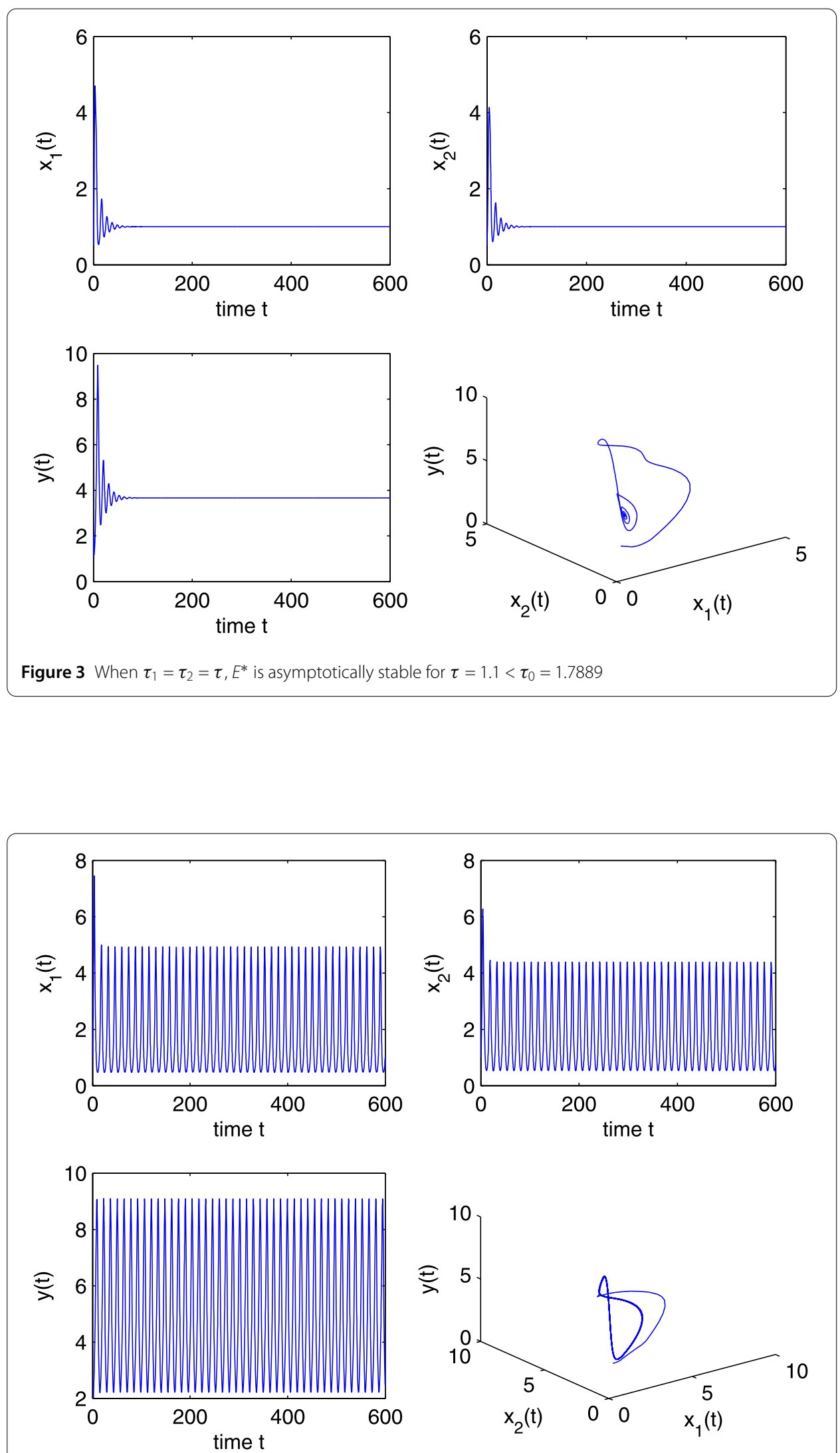

Figure 4 When $\tau_{1}=\tau_{2}=\tau$, $E^{*}$ undergoes a Hopf bifurcation for $\tau=1.8>\tau_{0}=1.7889$ 

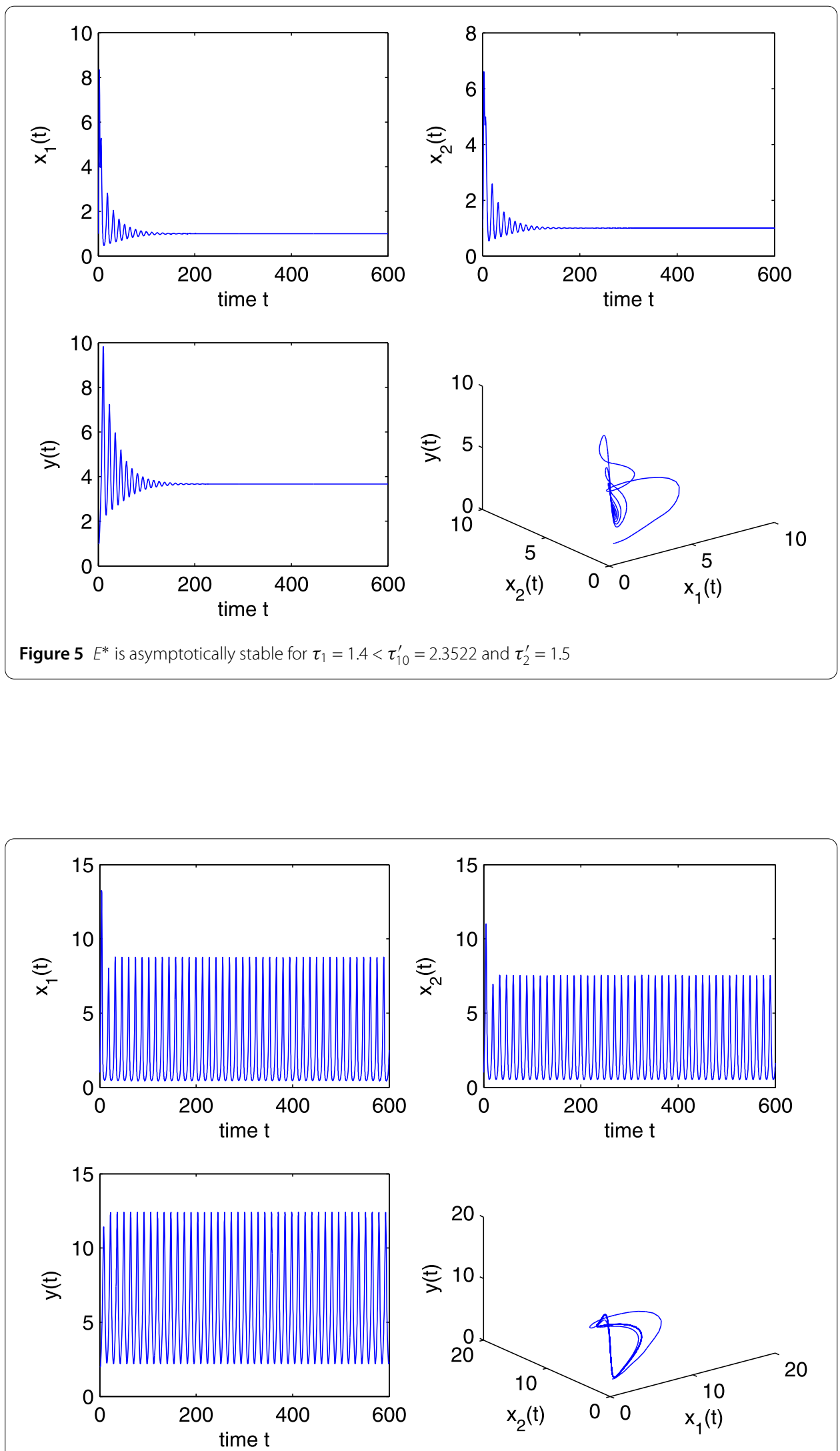

Figure $6 E^{*}$ undergoes a Hopf bifurcation for $\tau_{1}=2.4>\tau_{10}^{\prime}=2.3522$ and $\tau_{2}^{\prime}=1.5$ 
tain ecological significance and provides a powerful theoretical basis for the sustainable survival of natural populations.

In addition, stage structure for the prey is investigated in this paper due to the predator feeding only on the immature prey. If we were concerned with the combined effects of stage structure for both the predator and the prey, what will the dynamical behavior of the system be? This issue is left as our future research.

\author{
Acknowledgements \\ The authors would like to thank the referees for the careful reading of the manuscript and valuable suggestions. \\ Funding \\ This work was supported by the National Natural Science Foundation of China (No. 11472116), the Key Program of the \\ National Natural Science Foundation of China (No. 11632008), and Postgraduate Research \& Practice Innovation Program \\ of Jiangsu Province (KYCX17_1784).
}

\title{
Competing interests
}

The authors declare that they have no competing interests.

\section{Authors' contributions}

All authors contributed equally to the writing of this paper. The authors read and approved the final manuscript.

\section{Publisher's Note}

Springer Nature remains neutral with regard to jurisdictional claims in published maps and institutional affiliations.

Received: 21 February 2018 Accepted: 8 July 2018 Published online: 24 July 2018

\section{References}

1. Wangersky, P.J., Cunningham, W.J.: Time lag in prey-predator population models. Ecology 38(1), 136-139 (1957)

2. May, R.M.: Time delay versus stability in population models with two and three trophic levels. Ecology 54(2), 315-325 (1973)

3. Hu, G.P., Li, X.L.: Stability and Hopf bifurcation for a delayed predator-prey model with disease in the prey. Chaos Solitons Fractals 45(3), 229-237 (2012)

4. Banshidhar, S., Swarup, P.: Effects of supplying alternative food in a predator-prey model with harvesting. Appl. Math. Comput. 234, 150-166 (2014)

5. Yang, R.Z: Hopf bifurcation analysis of a delayed diffusive predator-prey system with nonconstant death rate. Chaos Solitons Fractals 81(6), 224-232 (2015)

6. Zhu, X.Y., Dai, Y.X., Li, Q.L., Zhao, K.H.: Stability and Hopf bifurcation of a modified predator-prey model with a time delay and square root response function. Adv. Differ. Equ. 2017, 235 (2017). https://doi.org/10.1186/s13662-017-1292-1

7. Li, X.H., Hou, J.Y: Bursting phenomenon in a piecewise mechanical system with parameter perturbation in stiffness. Int. J. Non-Linear Mech. 81, 165-176 (2016)

8. Li, X.H., Hou, J.Y., Chen, J.F.: An analytical method for Mathieu oscillator based on method of variation of parameter. Commun. Nonlinear Sci. Numer. Simul. 37, 326-353 (2016)

9. Chen, X.Y., Huang, L.H.: A Filippov system describing the effect of prey refuge use on a ratio-dependent predator-prey model. J. Math. Anal. Appl. 428(2), 817-837 (2015)

10. Peng, M., Zhang, Z.D., Wang, X.D.: Hybrid control of Hopf bifurcation in a Lotka-Volterra predator-prey model with two delays. Adv. Differ. Equ. 2017, 387 (2017). https://doi.org/10.1186/s13662-017-1434-5

11. Meng, X.Y., Huo, H.F., Zhang, X.B., Xiang, H.: Stability and Hopf bifurcation in a three-species system with feedback delays. Nonlinear Dyn. 64(4), 349-364 (2011)

12. Boonrangsiman, S., Bunwong, K., Moore, E.J.: A bifurcation path to chaos in a time-delay fisheries predator-prey model with prey consumption by immature and mature predators. Math. Comput. Simul. 124, 16-29 (2016)

13. Khajanchi, S.: Modeling the dynamics of stage-structure predator-prey system with Monod-Haldane type response function. Appl. Math. Comput. 302, 122-143 (2017)

14. Wang, X.D., Peng, M., Liu, X.Y.: Stability and Hopf bifurcation analysis of a ratio-dependent predator-prey model with two time delays and Holling type III functional response. Appl. Math. Comput. 268, 496-508 (2015)

15. Ruan, S., Wei, J.: On the zero of some transcendental functions with applications to stability of delay differential equations with two delays. Dyn. Contin. Discrete Impuls. Syst., Ser. A Math. Anal. 10(6), 863-874 (2003)

16. Song, Y., Wei, J.: Bifurcation analysis for Chen's system with delayed feedback and its application to control of chaos. Chaos Solitons Fractals 22(1), 75-91 (2004)

17. Hale, J.K.: Theory of Functional Differential Equations. Springer, New York (1977)

18. Hassard, B.D., Kazarinoff, N.D., Wan, Y.H.: Theory and Application of Hopf Bifurcation. Cambridge University Press, Cambridge (1981)

19. Zhang, Z.D., Bi, Q.S.: Bifurcation in a piecewise linear circuit with switching boundaries. Int. J. Bifurc. Chaos 22, 2 (2012). https://doi.org/10.1142/S0218127412500344 